\title{
Synergistic antitumor effects of histone deacetylase inhibitor scriptaid and bortezomib against ovarian cancer cells
}

\author{
KAROLINA JANYST, MICHAŁ JANYST, MARTA SIERNICKA and WITOLD LASEK \\ Department of Immunology, Centre of Biostructure Research, Medical University of Warsaw, 02-097 Warsaw, Poland
}

Received October 11, 2017; Accepted December 29, 2017

DOI: $10.3892 /$ or.2018.6248

\begin{abstract}
Despite debulking surgery and good initial response to chemotherapy, the majority of patients with advanced ovarian cancer relapse and succumb to their disease. Thus, there is a pressing need to improve treatment outcome. In the present study, the antitumor activity of histone deacetylase (HDAC) inhibitor scriptaid in combination with bortezomib or conventional chemotherapeutics was tested in vitro against representative ovarian cancer cell lines: SKOV-3, MDAH 2774 , and OVP-10. Incubation of ovarian cancer cells with scriptaid and bortezomib (or doxorubicin) led to synergistic antitumor effects. As shown in the Annexin V-FITC/PI assay and western blot analysis of caspase-3/-9 and p21 protein expression, these synergistic antitumor effects were due to both induction of apoptosis and inhibition of proliferation. Since synergistic antitumor activity of scriptaid and bortezomib appeared in suboptimal concentrations, one can assume that the administration of the combination of these agents to ovarian cancer patients can exert the therapeutic effect in parallel with limited general toxicity of the treatment.
\end{abstract}

\section{Introduction}

Ovarian cancer is the leading cause of death from gynecological cancers in highly developed countries, and responsible for approximately 14,000 deaths each year in the United States (1). The 5-year survival of patients with ovarian cancer ranges from 30 to $50 \%$. Despite debulking surgery and standard adjuvant chemotherapy, most patients with advanced disease relapse within 2 years after diagnosis (2). In accordance with these data, there is a pressing need for new approaches to the medical management of this disease. Understanding of the specific neoplastic process and the knowledge of cancer cell biology (3) will allow the identification and development of

Correspondence to: Professor Witold Lasek, Department of Immunology, Centre of Biostructure Research, Medical University of Warsaw, Banacha 1a, 'F' Bldg, 02-097 Warsaw, Poland

E-mail: witold.lasek@wum.edu.pl

Key words: ovarian cancer, scriptaid, doxorubicin, bortezomib, HDAC inhibitors novel biological agents in clinical oncology which could be used in addition to classical chemotherapy. This also applies to ovarian cancer in which genomic and epigenetic alterations have been frequently shown (4-6).

An interesting class of drugs targeting mechanisms strictly related to cancer initiation and progression are histone deacetylase (HDAC) inhibitors (7). These agents are involved in the regulation of apoptosis and differentiation of tumor cells, suppression of angiogenesis, cell migration and motility, and immunomodulation $(8,9)$. Eighteen mammalian HDAC enzymes have been identified to date, which can be subdivided into different families according to their homology with yeast HDACs (10). To date, 4 HDAC inhibitors have been approved in oncology (vorinostat, panobinostat, belinostat and romidepsin) and many other drugs from this group are currently being tested in clinical trials. Some HDAC inhibitors have been found to be effective in preclinical studies in ovarian carcinoma, either as monotherapy or in combination with other agents (11-14). There has been a strong rationale to test HDAC inhibitors as anticancer agents against ovarian cancer in the clinic (15-17). An interesting pan-HDAC inhibitor that has been poorly studied is scriptaid, 6-[1,3-dioxo- $1 \mathrm{H}, 3 \mathrm{H}$ benzo(de)isoquinolin-2-yl]-hexanoic acid hydroxyamide. This agent was shown to inhibit the growth and induce differentiation and/or apoptosis in breast, glioma, colon and also gynecological cancer cells (18-21). Some data suggest that the antitumor effectiveness of scriptaid can be strongly boosted, even synergistically, by its combination with other agents/ modalities (22).

Another group of agents that is being explored in experimental and clinical oncology are proteasome inhibitors (23). Blocking proteasome activity leads to accumulation of damaged proteins, resulting in caspase activation and cell death (23). Bortezomib is the first proteasome inhibitor approved by the US Food and Drug Administration for the treatment of relapsed/refractory multiple myeloma. Recent research demonstrated that combinations of HDAC3 inhibitor BG45 and bortezomib exerted a synergistic therapeutic effect in a murine xenograft model of human multiple melanoma (24). A combination of HDAC inhibitor vorinostat with bortezomib was found to improve survival in comparison with bortezomib alone in patients with multiple myeloma (25).

In the present study, we investigated the antitumor effects of scriptaid, used either alone or in combination with bortezomib, as well as with standard chemotherapeutics: 
paclitaxel, doxorubicin, carboplatin or etoposide, on representative ovarian cancer cells in vitro. The rationale for combining scriptaid together with bortezomib or with conventional chemotherapeutics was that the former can sensitize ovarian cancer cells and can reverse resistance of cancer cells to drugs used in currently established therapeutic protocols (26-29).

\section{Materials and methods}

Tumor cells and reagents. Three ovarian cancer cell lines were studied: SKOV-3 (cat. no. HTB-77; ATCC, Manassas, VA, USA), MDAH 2774 (cat. no. CRL-10303; ATCC), and OVP-10 (obtained from Dr Barbara Szaniawska, Department of Cell Biology, The Maria Skłodowska Curie Memorial Cancer Centre and Institute of Oncology, Warsaw, Poland). MDAH 2774 cells were cultured either in Dulbecco's MEM with $4.5 \mathrm{~g} / 1$ glucose, sodium pyruvate and L-glutamine (high glucose DMEM) (Corning, Corning, NY, USA) and OVP-10 and SKOV-3 cells in RPMI-1640 (Sigma-Aldrich, Irvine, UK) [supplemented with antibiotic-antimycotic (Corning), and $10 \%$ heat-inactivated fetal bovine serum (FBS) (Gibco BRL, Paisley, UK)]. The cells were maintained in $25-\mathrm{cm}^{2}$ tissue flasks (Nunc, Roskdile, Denmark) at $37^{\circ} \mathrm{C}$ in a humidified atmosphere of $5 \% \mathrm{CO}_{2}$ and were passaged two to three times weekly. The following antitumor agents were tested against ovarian cancer cells: doxorubicin (Pfizer), etoposide (Ebeve), paclitaxel (Actavis), carboplatin (Medac), scriptaid (Selleckchem), and bortezomib (Millenium Pharm. Inc.). The drugs were first diluted in DMSO or water for injection and then in growth medium.

MTT assay. The cytotoxic effect of scriptaid, bortezomib, and other antitumor chemotherapeutics on SKOV-3, MDAH 2774 and OVP-10 ovarian cancer cells was tested in a standard 3-(4,5-dimethylthiazol-2-yl)-2,5-diphenyltetrazolium bromide (MTT) assay. The cells were placed in 96-well plates (flat bottom) at a concentration of $12 \times 10^{3}$ (SKOV-3), or $4 \times 10^{3}$ (OVP-10, MDAH 2774) cells/well and drugs or solvent control were added for a 72-h exposure in a final volume of $0.2 \mathrm{ml} /$ well. At the end of the incubation, $25 \mu 1$ of MTT $(2.5 \mathrm{mg} / \mathrm{ml}$ stock solution) was added to each well. After a 4-h incubation with MTT, the cells were centrifuged ( $350 \mathrm{x} \mathrm{g}, 10 \mathrm{~min}$ ), supernatants were removed and formazan crystals were dissolved in $100 \mu \mathrm{l}$ of acid DMSO solution. The absorbance in each well was measured using an ELISA reader (Asys UVM 340 Microplate Reader, Biochrom Ltd., UK) using a 540-nm filter. The means \pm standard deviations (SDs) were determined for triplicate samples. The cytotoxic/cytostatic effect was expressed as the relative viability and was calculated according to the formula: Relative viability $=[$ (experimental absorbance - background absorbance)/(absorbance of vehicletreated cells - background absorbance) x $100 \%$.

Apoptosis assay by flow cytometry. Flow cytometric analysis was performed using propidium iodide (PI) and fluorescein isothiocyanate (FITC)-labeled Annexin V (eBioscience, Vienna, Austria). SKOV-3, MDAH 2774 or OVP-10 cells, after the incubation with agents for $72 \mathrm{~h}$, were washed with cold binding buffer at $4^{\circ} \mathrm{C}$ and centrifuged at $500 \mathrm{x}$ g for $5 \mathrm{~min}$. Then, cells were resuspended in binding buffer at a

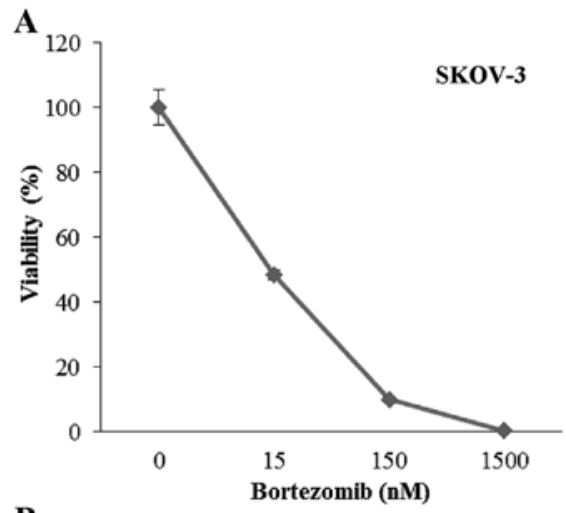

B
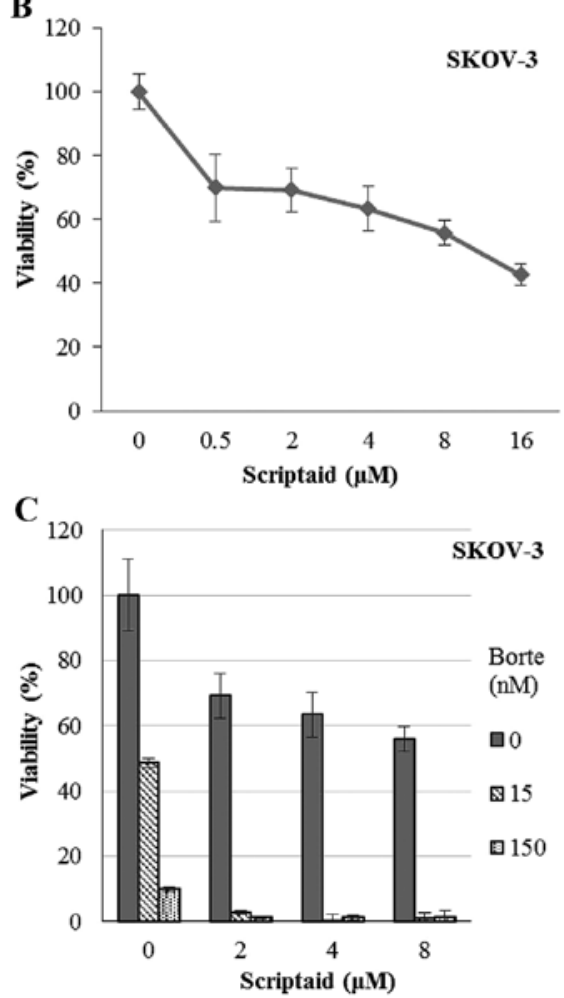

Figure 1. Inhibitory effects of different concentrations of scriptaid (A), bortezomib (Borte) (B), and both (C) on the viability of SKOV-3 cells. Cancer cells were incubated for $72 \mathrm{~h}$ with different concentrations of scriptaid or bortezomib and the cytotoxic effect of the agents was measured using an MTT assay. The data shown are the mean viability \pm SD of triplicates.

concentration of $4 \times 10^{6}$ cells $/ \mathrm{ml}$. Cell suspensions $\left(2 \times 10^{5}\right.$ cells in $50 \mu \mathrm{l}$ ) were mixed with $1 \mu \mathrm{l}$ FITC Annexin V and $1 \mu \mathrm{l}$ propidium iodide (PI) and incubated for $15 \mathrm{~min}$ at room temperature in the dark. Finally, the cells were analyzed by flow cytometry (BD Accuri ${ }^{\mathrm{TM}}$ C6 Plus flow cytometer).

Western blotting. At the end of the incubation with the agents $(72 \mathrm{~h})$, SKOV- 3 cells were washed three times with PBS, pelleted and lysed in RIPA buffer containing protease inhibitor cocktail and phosphatase inhibitor cocktail. The lysates were centrifuged at $15,000 \mathrm{rpm}$ for $30 \mathrm{~min}$ at $4^{\circ} \mathrm{C}$ and the supernatant was collected. The protein concentration in the lysates was determined by bicinchoninic acid assay (BCA assay). Next, the cell extracts were separated on $10 \%$ SDS-polyacrylamide gel (cleaved-caspase 9) or $12 \%$ SDS-polyacrylamide gel (p21 and procaspase 3). The primary antibodies (all from Cell Signaling, at 1:1,000 dilutions) were 
A

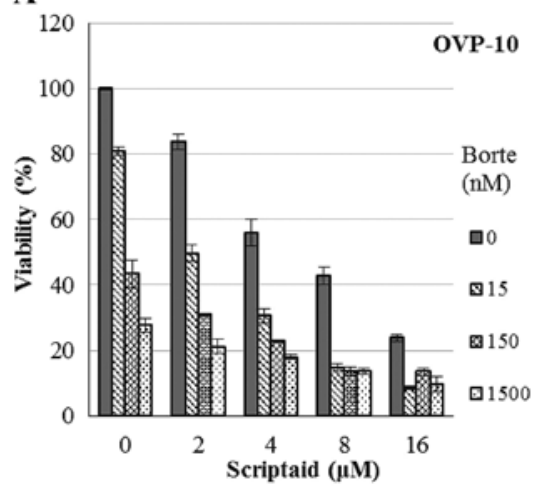

B

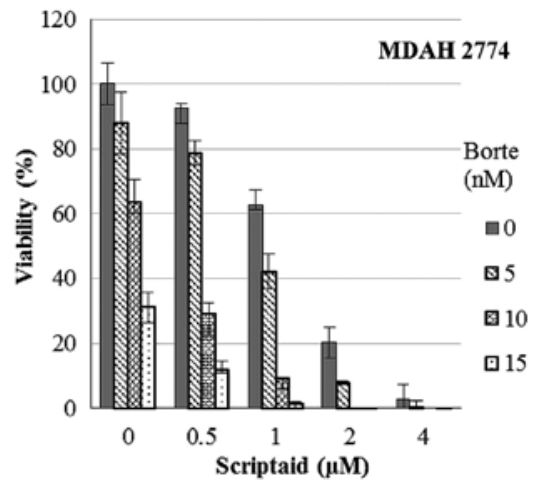

Figure 2. Inhibitory effects of different concentrations of scriptaid and bortezomib (Borte) on OVP-10 (A) and MDAH 2774 (B) ovarian carcinoma cells. Cancer cells were incubated for $72 \mathrm{~h}$ with different concentrations of scriptaid and bortezomib and the cytotoxic effect of the agents was measured using an MTT assay. The data shown are the mean viability \pm SD of triplicates.

used for the overnight incubation at $4^{\circ} \mathrm{C}$ in the presence of $5 \%$ non-fat dry milk. After washing with TBST, membranes were incubated with anti-rabbit HRP-coupled secondary antibody (Jackson ImmunoResearch) in 5\% dry milk in TBST for $1 \mathrm{~h}$ at room temperature. The expression of targeted proteins was detected with the enhanced chemiluminescent detection system and visualized with Stella 8300 bioimager (Raytest, Straubenhardt, Germany). The blots were re-probed with anti$\beta$-actin-peroxidase purified immunoglobulin (clone AC-15; Sigma-Aldrich) at 1:50,000 dilution for $45 \mathrm{~min}$.

Statistical analysis. The data in the figures are presented as the mean \pm SD. The combination index (CI) for drug interaction (synergism) was calculated using CompuSyn software (ComboSyn, Inc.) (30). According to Chou-Talalay method, if $\mathrm{CI}$ is $<1$, the two drugs show synergism; if CI is $>1$, the two drugs show antagonism (31).

\section{Results}

Synergistic inhibitory effects of scriptaid and bortezomib on the viability of SKOV-3, OVP-10, and MDAH 2774 cells. In our preliminary experiments, cytotoxic effects of single agents: scriptaid, bortezomib, doxorubicin, paclitaxel, carboplatin or etoposide were determined in a 72-h MTT assay against SKOV-3, OVP-10, and MDAH 2774 cells. A dose-dependent decrease in cell viability was observed in all cultures (data not shown). In the next step, combinations of scriptaid with

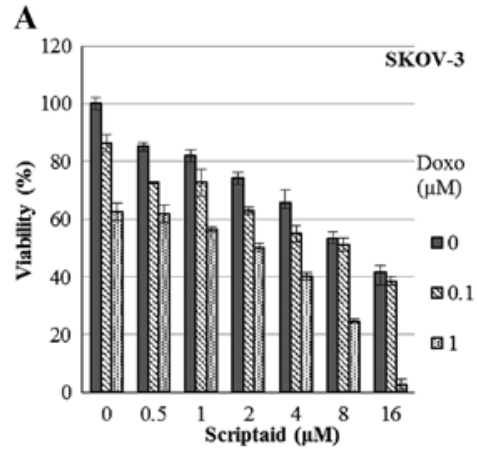

B

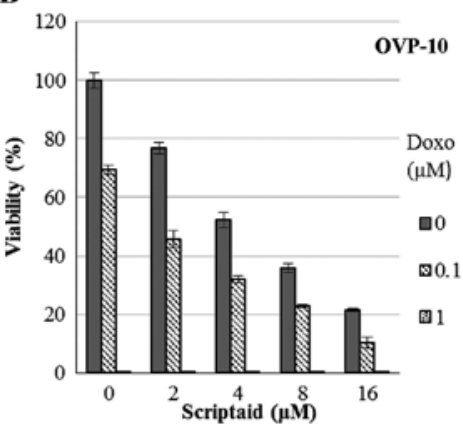

C

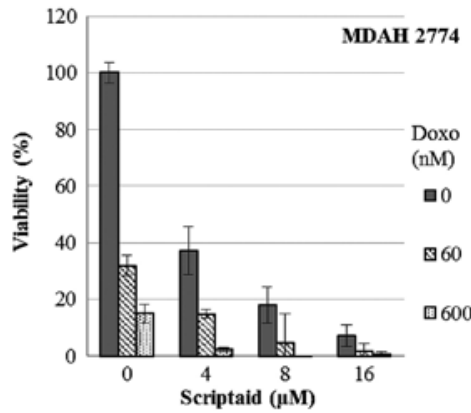

Figure 3. Inhibitory effects of different concentrations of scriptaid and doxorubicin (Doxo) on SKOV-3 (A), OVP-10 (B) and MDAH 2774 (C) ovarian carcinoma cells. Cancer cells were incubated for $72 \mathrm{~h}$ with different concentrations of scriptaid and bortezomib and the cytotoxic effect of the agents was measured using an MTT assay. The data shown are the mean viability \pm SD of triplicates.

other agents were tested. Incubation of SKOV-3 cells with scriptaid $(0.5-16 \mu \mathrm{M})$ or bortezomib $(15-1,500 \mathrm{nM})$ resulted in a dose-dependent decreasing viability (Fig. 1A and B). In fact, the highest concentrations of bortezomib completely killed SKOV-3 cells. The combination of scriptaid and bortezomib caused a synergistic effect $(\mathrm{CI}<1$, between 0.009 and 0.69$)$ and a concentration as low as $15 \mathrm{nM}$ of bortezomib and $2 \mu \mathrm{M}$ dose of scriptaid led to killing of almost all cells (Fig. 1C). Similar effects were observed in cultures of OVP-10 and MDAH 2774 ovarian cancer cells. Combination treatment induced stronger antitumor effect than the treatment with either scriptaid or bortezomib and resulted in a synergistic effect with $\mathrm{CI}$ between 0.335 and 0.819 (OVP-10) and between 0.183 and 0.917 (MDAH 2774) (Fig. 2).

Synergistic inhibitory effects of scriptaid and doxorubicin on the viability of SKOV-3, OVP-10, and MDAH 2774 cells. Paclitaxel or carboplatin, when used in combination with scriptaid, presented additive antitumor effects against ovarian cancer cells while etoposide did not significantly affect cell 


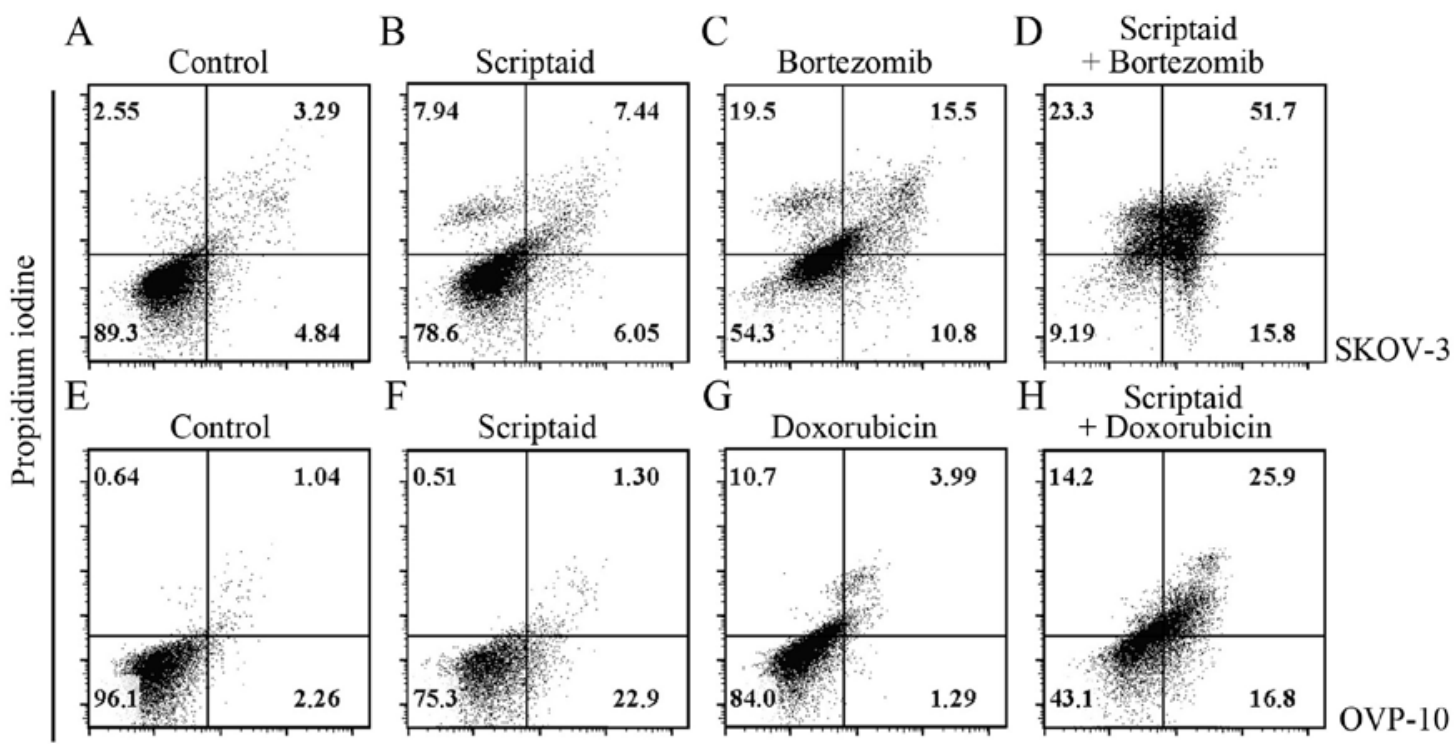

Annexin V

Figure 4. Detection of apoptotic SKOV-3 (A-D) and OVP-10 (E-H) cells by flow cytometry in cultures incubated with scriptaid + bortezomib and scriptaid + doxorubicin, respectively. Early apoptotic or late apoptotic and necrotic percentages were quantified by flow cytometry, as described in Materials and methods, and the results demonstrated that there were significant higher rates of early apoptosis or late apoptosis and necrosis in the combined treatment group in each case. (A) Control. (B) Scriptaid $(2 \mu \mathrm{M})$. (C) Bortezomib (15 nM). (D) Combination of bortezomib and scriptaid. (E) Control. (F) Scriptaid (2 $\mu \mathrm{M})$. (G) Doxorubicin $(50 \mathrm{nM})$. (H) Combination of doxorubicin and scriptaid.

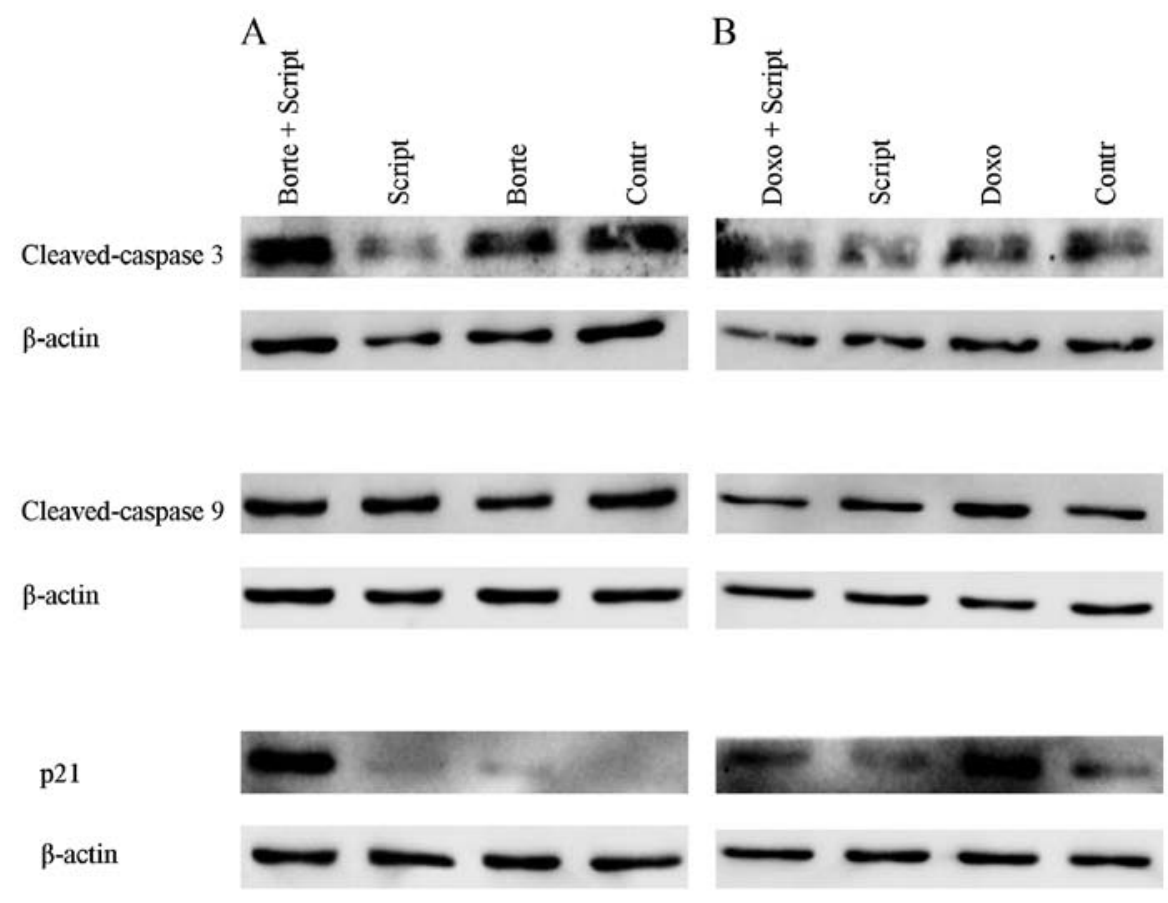

Figure 5. Western blot analysis of p21, cleaved-caspase 3, and cleaved-caspase 9 in SKOV-3 cells incubated (A) with bortezomib (Borte) (10 nM) and/or scriptaid $(1 \mu \mathrm{M})$ and (B) doxorubicin (Doxo) $(10 \mathrm{nM})$ and/or scriptaid $(2 \mu \mathrm{M})$ for $72 \mathrm{~h}$. $\beta$-actin was used as a control.

viability (data not shown). In contrast, joint administration of scriptaid (concentration range $0.5-16 \mu \mathrm{M}$ ) with doxorubicin $(60 \mathrm{nM}-1 \mu \mathrm{M})$ induced synergistic cytotoxic activity in cultures of SKOV-3, OVP-10 and MDAH 2774 ovarian cancer cell lines. In these cases, the combined treatment caused synergistic effects with a CI between 0.027 and 0.727 (SKOV-3), 0.059 and 0.456 (OVP-10) (Fig. 3A and B). In MDAH 2774 cells, a synergistic effect was observed in the combinations with the highest concentrations of scriptaid $(8-16 \mu \mathrm{M})$ with $\mathrm{CI}$ between 0.188 and 0.987 (Fig. 3C).

Effects of combined treatment on cellular apoptosis. To determine the effects of scriptaid, bortezomib, doxorubicin and combinations of these agents on cellular apoptosis of SKOV-3, 
OVP-10 and MDAH 2774 cells, Annexin V-FITC/PI apoptosis assay was used. Treatment with scriptaid $(0.5-16 \mu \mathrm{M})$, bortezomib (5-1,500 $\mathrm{nM})$ or doxorubicin $(0.06-10 \mu \mathrm{M})$ for $72 \mathrm{~h}$ induced a concentration-dependent increase in cellular apoptosis of each cell line (data not shown). Combined treatment with scriptaid and bortezomib or scriptaid and doxorubicin resulted in a significant increase in cellular apoptosis of cells when compared with the single agents and the control. Representative results are shown in Fig. 4. For example, in the SKOV-3 cell culture incubated with $2 \mu \mathrm{M}$ scriptaid $+15 \mathrm{nM}$ bortezomib, late apoptotic and dead cells constituted $51.7 \%$ of the cell population in comparison with 7.44, 15.5 and $3.29 \%$ in scriptaid, bortezomib, and control cultures, respectively (Fig. 4A-D). Only 9.19\% cells remained fully alive in a doubletreated culture (Fig. 4D). Similar effect - the lowest percentage of living cells was observed in culture of MDAH 2774 cells treated with both scriptaid and bortezomib (data not shown). Results of scriptaid and doxorubicin combination were very much alike. In the case of the OVP-10 ovarian cancer cells incubated with $2 \mu \mathrm{M}$ scriptaid $+50 \mathrm{nM}$ doxorubicin, the percentage of late apoptotic/dead cells was highest and the number of living cells was smallest, in comparison with the single agent-treated cultures (Fig. 4E-H).

Effects of the combined treatment on the expression of proapoptotic and cell cycle arrest proteins. HDAC inhibitors and bortezomib, as well as conventional antitumor chemotherapeutics, have been reported to regulate the level of different proteins that control the cell cycle and apoptosis $(26,32,33)$. In the present study, we analyzed the influence of scriptaid in combination with either bortezomib or doxorubicin on the expression levels of apoptotic proteins caspase 3 and 9, and the key marker of cell cycle arrest protein p21. As shown in Fig. 5, scriptaid, bortezomib, and doxorubicin, either alone or in combination, did not change expression of caspase 3 and 9 in the 72-h cultures of SKOV-3 cells. In contrast, treatment with scriptaid and bortezomib resulted in a marked increase in p21, suggesting that cell cycle arrest mechanisms significantly contributed to the cytotoxic/cytostatic effects of this combination.

\section{Discussion}

Over the last two decades, through improved surgical cytoreduction and more chemotherapy options, the survival of patients with advanced ovarian cancer and the chance to cure this disease have increased significantly (34). However, current conventional chemotherapy is non-selective and often results in a marked toxicity. Due to increased knowledge of the molecular, genetic and epigenetic background of ovarian cancer, novel treatment approaches are being investigated (5). Recent implementation of olaparib to the drug armamentarium used in ovarian cancer (to treat selected patients) is the example of such a successful search (35). Since epigenetic changes may play a role in the pathogenesis of many ovarian cancers, there have been studies focusing on the interference with histone modification and DNA methylation processes.

In the present study, we demonstrated unique combinations of the experimental agent, HDAC inhibitor scriptaid, with bortezomib (the proteasome inhibitor used in patients with hematological malignancies) or with conventional chemotherapeutics used to treat gynecological cancers. The most significant combination, scriptaid and bortezomib, was found to act synergistically against cancer cells of three representative ovarian cancer cell lines. Notably, a growth inhibitory effect of this combination was manifested at suboptimal concentrations of the single agents. This effect can be attributed both to induction of apoptosis (Fig. 4) and cell cycle inhibition (Fig. 5). Our results are in agreement with studies of other authors who tested various combinations of HDAC and proteasome inhibitors in colon (36), hepatocellular (32), and lung (26) cancer models in vitro.

HDAC inhibitors (including scriptaid) are recognized as promising drugs in oncology, since they frequently reverse epigenetic changes in different types of tumors (7). It was recently shown that there are other proteins, in addition to histones, whose activity is affected by acetylation (9). Four HDAC inhibitors have been approved in clinical oncology to date: vorinostat, panobinostat, belinostat (hydroxamate-based pan-HDAC inhibitor), and romidepsin (cyclic tetrapeptide HDAC inhibitor). All these drugs are used in hematological malignancies. However, despite their evident benefit in lymphoma and myeloma, these drugs have not been found effective in studies with different solid tumors, including ovarian cancer $(16,37)$. The general view is that HDAC inhibitors are promising drugs in solid malignancies but only when combined with other anticancer drugs/agent or radiotherapy $(7,38)$.

Scriptaid is an HDAC inhibitor that was identified by screening a library of 16,320 compounds (DIVERSet; Chembridge, San Diego, CA, USA) in 2000 (39). The advantage of this agent over other known HDAC inhibitors is its relative non-toxic effect on normal cells $(19,21)$, and possible preferential activity against gynecological cancers $(18,21,40)$. This was one of the rationales for selecting scriptaid in our study using combination treatment with proteasome inhibitor bortezomib and other chemotherapeutics on ovarian cancer cell lines. We were additionally encouraged by the observation of the synergistic antitumor and chemosensitization effect of scriptaid and various proteasome inhibitors on human colorectal cancer cells (41) and antitumor effects (in renal cancer model in mice) of the combination of pan-HDAC inhibitor vorinostat (SAHA) with bortezomib (42). Furthermore, vorinostat and bortezomib inhibited tumor growth in a prostate tumor model in mice (43). Of note, there have been numerous clinical trials to improve the treatment of myeloma (and also lymphoma), in which bortezomib is approved as a drug, by combining HDAC inhibitors vorinostat or panobinostat with different proteasome inhibitors $(25,44,45)$. Recently, the synergistic activity of HDAC inhibitor trichostatin (TSA) and bortezomib against taxan-resistant ovarian cancer cell lines has been reported (46). Since scriptaid has been found less toxic than TSA, the former seems to be more suitable for further in vivo investigation of combination protocols.

In conclusion, our data suggest that the use of scriptaid may enhance the effectiveness of conventional chemotherapy of ovarian cancer and that the new combination: scriptaid + bortezomib is worth considering as a treatment option for heavily pretreated patients. This combination may be favorable, by analogy to olaparib (35), in a selected group of patients, for 
example in protocols of intraperitoneal administration in women with recurrent disease. Encouraging results of a recent phase I trial of intraperitoneal treatment of bortezomib in combination with carboplatin in patients with persistent/recurrent ovarian cancer argue for this assumption (47).

\section{Acknowledgements}

The authors are grateful to Drs Małgorzata Firczuk and Małgorzata Wańczyk for providing various reagents.

\section{Competing interests}

The authors declare that they have no competing interests.

\section{References}

1. Siegel RL, Miller KD and Jemal A: Cancer statistics, 2016. CA Cancer J Clin 66: 7-30, 2016.

2. Giornelli GH: Management of relapsed ovarian cancer: A review. Springerplus 5: 1197, 2016.

3. Bast RC Jr, Hennessy B and Mills GB: The biology of ovarian cancer: New opportunities for translation. Nat Rev Cancer 9: 415-428, 2009

4. Dong A, Lu Y and Lu B: Genomic/epigenomic alterations in ovarian carcinoma: Translational insight into clinical practice. J Cancer 7: 1441-1451, 2016.

5. Grunewald T and Ledermann JA: Targeted therapies for ovarian cancer. Best Pract Res Clin Obstet Gynaecol 41: 139-152, 2017.

6. Smith HJ, Straughn JM, Buchsbaum DJ and Arend RC: Epigenetic therapy for the treatment of epithelial ovarian cancer: A clinical review. Gynecol Oncol Rep 20: 81-86, 2017.

7. Eckschlager T, Plch J, Stiborova M and Hrabeta J: Histone deacetylase inhibitors as anticancer drugs. Int J Mol Sci 18: E1414, 2017.

8. Manal M, Chandrasekar MJ, Gomathi Priya J and Nanjan MJ: Inhibitors of histone deacetylase as antitumor agents: A critical review. Bioorg Chem 67: 18-42, 2016.

9. Mottamal M, Zheng S, Huang TL and Wang G: Histone deacetylase inhibitors in clinical studies as templates for new anticancer agents. Molecules 20: 3898-3941, 2015.

10. Ropero S and Esteller M: The role of histone deacetylases (HDACs) in human cancer. Mol Oncol 1: 19-25, 2007.

11. Budman DR, Tai J, Calabro A and John V: The histone deacetylase inhibitor panobinostat demonstrates marked synergy with conventional chemotherapeutic agents in human ovarian cancer cell lines. Invest New Drugs 29: 1224-1229, 2011.

12. Chao H, Wang L, Hao J, Ni J, Chang L, Graham PH, Kearsley JH and Li Y: Low dose histone deacetylase inhibitor, LBH589, potentiates anticancer effect of docetaxel in epithelial ovarian cancer via PI3K/Akt pathway in vitro. Cancer Lett 329: 17-26, 2013.

13. Cooper AL, Greenberg VL, Lancaster PS, van Nagell JR Jr, Zimmer SG and Modesitt SC: In vitro and in vivo histone deacetylase inhibitor therapy with suberoylanilide hydroxamic acid (SAHA) and paclitaxel in ovarian cancer. Gynecol Oncol 104: 596-601, 2007.

14. Konstantinopoulos PA, Wilson AJ, Saskowski J, Wass E and Khabele D: Suberoylanilide hydroxamic acid (SAHA) enhances olaparib activity by targeting homologous recombination DNA repair in ovarian cancer. Gynecol Oncol 133: 599-606, 2014.

15. Cassier PA, Floquet A, Penel N, Derbel O, Bui N'guyen B, Guastalla JP, Pissaloux D, Treilleux I, Saba CE, Blay JY, et al: The histone deacetylase inhibitor panobinostat is active in patients with advanced pretreated ovarian sex-cord tumors. Ann Oncol 25: 1074-1075, 2014.

16. Matulonis U, Berlin S, Lee H, Whalen C, Obermayer E, Penson R, Liu J, Campos S, Krasner C and Horowitz N: Phase I study of combination of vorinostat, carboplatin, and gemcitabine in women with recurrent, platinum-sensitive epithelial ovarian, fallopian tube, or peritoneal cancer. Cancer Chemother Pharmacol 76: 417-423, 2015.

17. Modesitt SC, Sill M, Hoffman JS and Bender DP; Gynecologic Oncology Group: A phase II study of vorinostat in the treatment of persistent or recurrent epithelial ovarian or primary peritoneal carcinoma: A Gynecologic Oncology Group study. Gynecol Oncol 109: 182-186, 2008.
18. Giacinti L, Giacinti C, Gabellini C, Rizzuto E, Lopez M and Giordano A: Scriptaid effects on breast cancer cell lines. J Cell Physiol 227: 3426-3433, 2012.

19. Janaki Ramaiah M, Naushad SM, Lavanya A, Srinivas C, Anjana Devi T, Sampathkumar S, Dharan DB and Bhadra MP: Scriptaid cause histone deacetylase inhibition and cell cycle arrest in HeLa cancer cells: A study on structural and functional aspects. Gene 627: 379-386, 2017.

20. Sharma V, Koul N, Joseph C, Dixit D, Ghosh S and Sen E: HDAC inhibitor, scriptaid, induces glioma cell apoptosis through JNK activation and inhibits telomerase activity. J Cell Mol Med 14: 2151-2161, 2010.

21. Takai N, Ueda T, Nishida M, Nasu K and Narahara H: A novel histone deacetylase inhibitor, Scriptaid, induces growth inhibition, cell cycle arrest and apoptosis in human endometrial cancer and ovarian cancer cells. Int J Mol Med 17: 323-329, 2006

22. Berghauser Pont LM, Kleijn A, Kloezeman JJ, van den Bossche W, Kaufmann JK, de Vrij J, Leenstra S, Dirven CM and Lamfers ML: The HDAC inhibitors scriptaid and LBH589 combined with the oncolytic virus Delta24-RGD exert enhanced anti-tumor efficacy in patient-derived glioblastoma cells. PLoS One 10: $\mathrm{e} 0127058,2015$.

23. Manasanch EE and Orlowski RZ: Proteasome inhibitors in cancer therapy. Nat Rev Clin Oncol 14: 417-433, 2017.

24. Minami J, Suzuki R, Mazitschek R, Gorgun G, Ghosh B, Cirstea D, Hu Y, Mimura N, Ohguchi H, Cottini F, et al: Histone deacetylase 3 as a novel therapeutic target in multiple myeloma. Leukemia 28: 680-689, 2014.

25. Dimopoulos M, Siegel DS, Lonial S, Qi J, Hajek R, Facon T, Rosinol L, Williams C, Blacklock H, Goldschmidt $\mathrm{H}$, et al: Vorinostat or placebo in combination with bortezomib in patients with multiple myeloma (VANTAGE 088): A multicentre, randomised, double-blind study. Lancet Oncol 14: 1129-1140, 2013.

26. Karthik S, Sankar R, Varunkumar K and Ravikumar V: Romidepsin induces cell cycle arrest, apoptosis, histone hyperacetylation and reduces matrix metalloproteinases 2 and 9 expression in bortezomib sensitized non-small cell lung cancer cells. Biomed Pharmacother 68: 327-334, 2014.

27. Ma YY, Lin H, Moh JS, Chen KD, Wang IW, Ou YC, You YS and Lung CC: Low-dose LBH589 increases the sensitivity of cisplatin to cisplatin-resistant ovarian cancer cells. Taiwan J Obstet Gynecol 50: 165-171, 2011.

28. Ong PS, Wang XQ, Lin HS, Chan SY and Ho PC: Synergistic effects of suberoylanilide hydroxamic acid combined with cisplatin causing cell cycle arrest independent apoptosis in platinumresistant ovarian cancer cells. Int J Oncol 40: 1705-1713, 2012.

29. Pradhan S, Mahajan D, Kaur P, Pandey N, Sharma C and Srivastava T: Scriptaid overcomes hypoxia-induced cisplatin resistance in both wild-type and mutant p53 lung cancer cells. Oncotarget 7: 71841-71855, 2016.

30. Chou TC and Talalay P: Quantitative analysis of dose-effect relationships: The combined effects of multiple drugs or enzyme inhibitors. Adv Enzyme Regul 22: 27-55, 1984.

31. Chou TC: Theoretical basis, experimental design, and computerized simulation of synergism and antagonism in drug combination studies. Pharmacol Rev 58: 621-681, 2006.

32. Spratlin JL, Pitts TM, Kulikowski GN, Morelli MP, Tentler JJ, Serkova NJ and Eckhardt SG: Synergistic activity of histone deacetylase and proteasome inhibition against pancreatic and hepatocellular cancer cell lines. Anticancer Res 31: 1093-1103, 2011.

33. Zhang H, Dong L, Chen Q, Kong L, Meng B, Wang H, Fu K, Wang X, Pan-Hammarström Q, Wang P, et al: Synergistic antitumor effect of histone deacetylase inhibitor and Doxorubicin in peripheral T-cell lymphoma. Leuk Res 56: 29-35, 2017.

34. Jessmon P, Boulanger T,Zhou W and Patwardhan P: Epidemiology and treatment patterns of epithelial ovarian cancer. Expert Rev Anticancer Ther 17: 427-437, 2017.

35. Meehan RS and Chen AP: New treatment option for ovarian cancer: PARP inhibitors. Gynecol Oncol Res Pract 3: 3, 2016.

36. Pitts TM, Morrow M, Kaufman SA, Tentler JJ and Eckhardt SG: Vorinostat and bortezomib exert synergistic antiproliferative and proapoptotic effects in colon cancer cell models. Mol Cancer Ther 8: 342-349, 2009.

37. Modesitt SC and Parsons SJ: In vitro and in vivo histone deacetylase inhibitor therapy with vorinostat and paclitaxel in ovarian cancer models: Does timing matter? Gynecol Oncol 119: 351-357, 2010.

38. Grassadonia A, Cioffi P, Simiele F, Iezzi L, Zilli M and Natoli C: Role of hydroxamate-based histone deacetylase inhibitors (Hb-HDACIs) in the treatment of solid malignancies. Cancers (Basel) 5: 919-942, 2013. 
39. Su GH, Sohn TA, Ryu B and Kern SE: A novel histone deacetylase inhibitor identified by high-throughput transcriptional screening of a compound library. Cancer Res 60: 3137-3142, 2000.

40. Keen JC, Yan L, Mack KM, Pettit C, Smith D, Sharma D and Davidson NE: A novel histone deacetylase inhibitor, scriptaid, enhances expression of functional estrogen receptor alpha (ER) in ER negative human breast cancer cells in combination with 5-aza 2'-deoxycytidine. Breast Cancer Res Treat 81: 177-186, 2003.

41. Abaza MS, Bahman AM, Al-Attiyah RJ and Kollamparambil AM: Synergistic induction of apoptosis and chemosensitization of human colorectal cancer cells by histone deacetylase inhibitor, scriptaid, and proteasome inhibitors: Potential mechanisms of action. Tumour Biol 33: 1951-1972, 2012.

42. Sato A, Asano T, Ito K, Sumitomo M and Asano T: Suberoylanilide hydroxamic acid (SAHA) combined with bortezomib inhibits renal cancer growth by enhancing histone acetylation and protein ubiquitination synergistically. BJU Int 109: 1258-1268, 2012.

43. Sato A, Asano T, Ito K and Asano T: Vorinostat and bortezomib synergistically cause ubiquitinated protein accumulation in prostate cancer cells. J Urol 188: 2410-2418, 2012.
44. Chhabra S: Novel proteasome inhibitors and histone deacetylase inhibitors: Progress in myeloma therapeutics. Pharmaceuticals (Basel) 10: 40, 2017.

45. Tan D, Diong CP, Loh Y and Goh YT: Histone deacetylase (HDAC) inhibitors when combined with a proteasome inhibitor are safe and effective in patients with extranodal natural killer/Tcell lymphoma (ENKTL). Ann Oncol 27: 1811-1812, 2016.

46. Jin X, Fang Y, Hu Y, Chen J, Liu W, Chen G, Gong M, Wu P, Zhu T, Wang S, et al: Synergistic activity of the histone deacetylase inhibitor trichostatin A and the proteasome inhibitor PS-341 against taxane-resistant ovarian cancer cell lines. Oncol Lett 13: 4619-4626, 2017.

47. Jandial DA, Brady WE, Howell SB, Lankes HA, Schilder RJ, Beumer JH, Christner SM, Strychor S, Powell MA, Hagemann AR, et al: A phase I pharmacokinetic study of intraperitoneal bortezomib and carboplatin in patients with persistent or recurrent ovarian cancer: An NRG Oncology/Gynecologic Oncology Group study. Gynecol Oncol 145: 236-242, 2017. 